\title{
Molecular characterization and serodiagnostic potential of Echinococcus granulosus hexokinase
}

Qi Xin ${ }^{1}$, Miaomiao Yuan ${ }^{1,2}$, Wei Lv ${ }^{1}$, Huanping Li ${ }^{1}$, Xiaoxia Song ${ }^{1}$, Jun $\mathrm{Lu}^{1}$ and Tao Jing ${ }^{{ }^{*}}$

\begin{abstract}
Background: Cystic echinococcosis (CE), caused by the larval stage of Echinococcus granulosus (sensu stricto), is a life-threatening but neglected zoonosis. Glycolytic enzymes are crucial molecules for the survival and development of E. granulosus. The aim of this study was to investigate the molecular characterization, immunogenicity, tissue distribution and serodiagnostic potential of $E$. granulosus hexokinase (EgHK), the first key enzyme in the glycolytic pathway.

Methods: EgHK was cloned and expressed in Escherichia coli. Specific serum antibodies were evaluated in mice immunized with recombinant EgHK (rEgHK). The location of EgHK in the larval stage of E. granulosus was determined using fluorescence immunohistochemistry, and the potential of $r E g H K$ as a diagnostic antigen was investigated in patients with CE using indirect enzyme-linked immunosorbent assay (ELISA).

Results: Recombinant EgHK could be identified in the sera of patients with CE and in mouse anti-rEgHK sera. High titers of specific immunoglobulin $\mathrm{G}$ were induced in mice after immunization with $\mathrm{rEgHK}$. EgHK was mainly located in the tegument, suckers and hooklets of protoscoleces and in the germinal layer and laminated layer of the cyst wall. The sensitivity and specificity of the rEgHK-ELISA reached $91.3 \%$ (42/46) and 87.8\% (43/49), respectively.

Conclusions: We have characterized the sequence, structure and location of EgHK and investigated the immunoreactivity, immunogenicity and serodiagnostic potential of $\mathrm{rEgHK}$. Our results suggest that EgHK may be a promising candidate for the development of vaccines against E. granulosus and an effective antigen for the diagnosis of human CE.
\end{abstract}

Keywords: Echinococcus granulosus, Hexokinase, Immunolocalization, Immunogenicity, Indirect ELISA, Diagnosis

\section{Background}

Cystic echinococcosis (CE) is caused by the larval stage of Echinococcus granulosus (sensu stricto) and occurs globally in livestock husbandry areas of South America, North Africa, Australia, western, central and eastern Europe and central Asia, particularly in western China [1]. CE is a life-threatening but neglected zoonosis,

*Correspondence: jtao@lzu.edu.cn

${ }^{1}$ Institute of Pathogenic Biology, School of Basic Medical Sciences, Lanzhou University, Lanzhou 730000, Gansu, People's Republic of China

Full list of author information is available at the end of the article especially in developing countries, that causes severe disorders of serious public health and economic concern [2]. A global estimate suggests that at least 50 million humans are infected with $E$. granulosus, with approximately more than 170,000 new cases every year $[3,4]$, resulting in an estimated 285,000 (95\% confidence interval: 218,515$366,133)$ disability-adjusted life years lost per annum and an annual economic loss of approximately US $\$ 3$ billion [3].

Similar to other parasites, the larval stage of $E$. granulosus obtains glucose from their hosts as their energy source. Analysis of E. granulosus genome data 
demonstrated that E. granulosus have complete pathways for both glycolysis and the tricarboxylic acid cycle during infection [5], while glycolysis is the main pathway for E. granulosus to generate energy and the vital intermediate products for physiological metabolism [6, 7]. Clearly, glycolytic enzymes play a crucial role in E. granulosus survival. To date, various glycolytic enzymes of E. granulosus, such as fructose-bisphosphate aldolase, enolase [8] and triosephosphate isomerase [9], have been identified in the tegument and parenchyma tissue of the parasite; these show antigenic properties and potential multifuncionality in E. granulosus. Thus, glycolytic enzymes represent promising targets for the development of both immune and drug intervention measures against echinococcosis.

Hexokinase (HK) (ATP: D-hexose-6-phosphotransferase, EC 2.7.1.1.) is an enzyme that facilitates the first step in glycolysis and catalyzes the phosphorylation of glucose to produce glucose 6-phosphate. HK is an important enzyme of glycolysis [10], and HK isozymes are widely distributed in a wide variety of species, ranging from plants, microbes, parasites to mammals including humans. The characteristics and functions of HK have been well examined in many parasites to date, including Plasmodium falciparum [11-13], Leishmania mexicana [14], Trypanosoma brucei $[15,16]$, Trypanosoma cruzi [17-19], Haemonchus contortus [20], Brugia malayi [21], Schistosoma mansoni [22-24] and Clonorchis sinensis $[25,26]$, but available information on HK from E. granulosus $(E g \mathrm{HK})$ is still limited.

In this study, the sequence and structure of $E g \mathrm{HK}$ were analyzed. A recombinant $E g \mathrm{HK}(\mathrm{r} E g \mathrm{HK})$ was expressed and the location of $E g \mathrm{HK}$ in the larval stage of E. granulosus was determined using fluorescence immunohistochemistry. Additionally, the serodiagnostic potential of the $\mathrm{r} E g \mathrm{HK}$ was investigated in patients with CE. Our results represent the first experimental data of $H K$ in $E$. granulosus and provide the foundation for further studies on $E g \mathrm{HK}$ in the framework of the diagnosis and prevention of human CE.

\section{Methods}

\section{Ethics statement}

Animal care and management procedures were conducted in compliance with the guidelines of the Institutional Animal Caring and Using Committee of Lanzhou University. Animals had free access to water and commercial mouse chow throughout the study. The experiment involving human participants was approved by the Human Research Ethics Committee of Lanzhou University. Each participant was provided with an explanation of the nature of the research and the study protocol, following which they all signed the informed consents.

\section{Bioinformatic analysis}

The complementary DNA (cDNA) sequence encoding $E g \mathrm{HK}$ was obtained from the cDNA library of adult E. granulosus constructed by our laboratory. The DNA sequence translations and the predictions of protein molecular mass, isoelectric point (pI), conserved domains and protein properties of $E g H \mathrm{H}$ were performed with ExPASy (http://au.expasy.org/tool/pi-tool.html). The TMHMM Server v.2.0 (http://www.cbs.dtu.dk/servi ces/TMHMM/) was used to predict the transmembrane domain and active center. SignalP 5.0 (http://www.cbs. dtu.dk/services/SignalP/) and SecretomeP 2.0a (http:// www.cbs.dtu.dk/services/SecretomeP/) were used to predict the signal peptide. The B-cell linear epitopes were analyzed using BepiPred software (version 1.0). The amino acid sequences of HK from different species were obtained from the GenBank and GeneDB databases. Multiple sequence alignment was performed using Clustal X software (version 2.0). The phylogenetic tree was constructed by the neighbor-joining method using MEGA software (version 7).

\section{Construction, expression and purification of $\mathrm{rEgHK}$}

The forward and backward primers were designed according to the open reading frame of the full-length nucleotide sequence of $E g \mathrm{HK}$. The coding sequence of $E g H K$ was PCR-amplified using the forward primers GAGGATCCATGGGGGTGCAATTC and backward primers CAGTCGACCTAGCCCGCGAAAAC with the BamHI and Sall restriction enzyme sites (underlined), respectively. The generated fragments were directionally inserted into the corresponding multiple cloning sites of pET30a(+) to construct plasmids encoding EgHK. After verification of the DNA inserts by sequencing, the recombinant plasmids were transformed into Escherichia coli strain BL21 (DE3) for the expression of $E g \mathrm{HK}$. After induction with $0.2 \mathrm{mM}$ isopropyl $\beta$-Dthiogalactopyranoside for $7 \mathrm{~h}$ at $20^{\circ} \mathrm{C}$, the cells were harvested and resuspended in a buffer containing $50 \mathrm{mM}$ $\mathrm{NaH}_{2} \mathrm{PO}_{4}$ and $300 \mathrm{mM} \mathrm{NaCL}$ and lysed by sonication. The samples were then sedimented by centrifugation at $12,000 \mathrm{~g}$ for $10 \mathrm{~min}$ at $4{ }^{\circ} \mathrm{C}$ to collect the inclusion bodies which were subsequently dissolved in solubilization buffer ( $8 \mathrm{M}$ urea and $50 \mathrm{mM}$ Tris, $\mathrm{pH} 8.0$ ) at a ratio of $1: 10(\mathrm{w} / \mathrm{v})$ for $30 \mathrm{~min}$ at $4{ }^{\circ} \mathrm{C}$ and then refolded by gradient dialysis for $60 \mathrm{~h}$ at $4{ }^{\circ} \mathrm{C}$. The purification of the his-tagged $\mathrm{r} E g \mathrm{HK}$ was performed using a $\mathrm{Ni}^{2+}$ affinity chromatography column (Qiagen, Hilden, Germany), and the molecular mass and purity of the purified proteins were assessed by $12 \%(\mathrm{v} / \mathrm{v})$ sodium dodecyl sulfate-polyacrylamide gel electrophoresis (SDS-PAGE). The protein concentration was determined with a BCA Protein Assay Kit (Beyotime, Shanghai, China). 


\section{Immunization of mice with rEgHK}

Eight-week-old specific pathogen-free female BALB/c mice $(18-20 \mathrm{~g})$ were obtained from the Laboratory Animal Center of Lanzhou University. The mice were immunized subcutaneously with $50 \mu \mathrm{g}$ purified $\mathrm{r} E g \mathrm{HK}$ emulsified in an equal volume of complete Freund's adjuvant (Sigma-Aldrich, St. Louis, MO, USA), followed up by two boosts with $50 \mu \mathrm{g}$ protein in an equal volume of incomplete Freund's adjuvant (Sigma-Aldrich) at 2 -week intervals. The mice in the control group received the same inoculation as the $\mathrm{r} E g \mathrm{HK}$-immunized group with only difference in the process being the replacement of $\mathrm{rEgHK}$ with phosphate buffered saline (PBS). The immune sera were then collected and identified by enzyme-linked immunosorbent assay (ELISA) for antibody titer, using horseradish peroxidase-conjugated goat anti-mouse immunoglobulin G (IgG).

\section{Western blotting}

A $10-\mu g$ aliquot of purified $\mathrm{r} E g \mathrm{HK}$ were transferred from the 12\% SDS-PAGE gel to a PVDF membrane. The membrane was blocked with 5\% skim milk in Tris-buffer saline-Tween 20 (TBST) overnight and then incubated with mouse anti-r $E g H K$ sera, sera collected from patients with $\mathrm{CE}$, sera collected from healthy subjects or preimmune mouse sera, at a dilution of $1: 200$ at $4{ }^{\circ} \mathrm{C}$ overnight. After two washes with TBS, the membrane was incubated with horseradish peroxidase-conjugated goat anti-mouse IgG or goat anti-human IgG (1:2000 dilution) (Sigma-Aldrich) at $37^{\circ} \mathrm{C}$ for $1 \mathrm{~h}$. The immunoblots were detected using an Enhanced Chemiluminescence Substrate Kit (Thermo Fisher Scientific, Waltham, MA, USA).

\section{Indirect immunofluorescence assays}

Echinococcus granulosus protoscoleces and cyst walls were isolated aseptically from hydatid cysts removed from the liver of infected sheep slaughtered in an abattoir (Xining, Qinghai, China) and fixed in $4 \%$ paraformaldehyde for $20 \mathrm{~h}$. Cyst walls were embedded in paraffin, sliced into $3-\mu \mathrm{m}$-thick sections, deparaffinized in xylene, dehydrated in ethanol and then incubated with $0.01 \mathrm{M}$ citrate buffer at $95^{\circ} \mathrm{C}$ for $30 \mathrm{~min}$ for thermal remediation. After three washes with PBS, the tissues were blocked with $5 \%$ bovine serum albumin (BSA) in PBS and treated with mouse anti-r $E g \mathrm{HK}$ sera or pre-immune mouse sera at a dilution of $1: 200$, at $37{ }^{\circ} \mathrm{C}$ for $1 \mathrm{~h}$, respectively, following which fluorescein isothiocyanate-conjugated goat anti-mouse IgG (1:200 dilution) (Sigma-Aldrich) was added and incubated at $37^{\circ} \mathrm{C}$ for a further $1 \mathrm{~h}$. Fluorescence was detected and images were acquired on an immunofluorescence microscope (model IX71; Olympus Corp., Tokyo, Japan ).

\section{rEgHK ELISA}

Sera from patients with CE (46 samples) and patients with Taenia solium cysticercosis (26 samples) were obtained from the Institute of Pathogenic Biology, Lanzhou University, China. Sera from healthy students (23 samples), collected at Lanzhou University, were used as a control group. Microplates were coated with $100 \mu \mathrm{L} /$ well of $\mathrm{r} E g \mathrm{HK}$ at $5 \mu \mathrm{g} / \mathrm{ml}$ in coating buffer overnight at $4{ }^{\circ} \mathrm{C}$. After three washes with PBST, the microplates were incubated with $1 \% \mathrm{BSA}$ at $37^{\circ} \mathrm{C}$ for $1 \mathrm{~h}$ for blocking. The serum samples were then added at 1:200 dilution in 1\% BSA in PBS-Tween (PBST) and incubated at $37^{\circ} \mathrm{C}$ for 1 $h$. The second antibody (horseradish peroxidase-conjugated goat anti-human IgG; Sigma-Aldrich) was diluted 1:5000 in 1\% BSA in PBST and incubated at $37^{\circ} \mathrm{C}$ for $1 \mathrm{~h}$. Subsequently, TMB substrate buffer was added $(100 \mu \mathrm{L} /$ well) and incubated at $37{ }^{\circ} \mathrm{C}$ for $15 \mathrm{~min}$. Finally, the reaction was terminated with $2 \mathrm{M} \mathrm{H}_{2} \mathrm{SO}_{4}(50 \mu \mathrm{L} /$ well $)$ and the absorbance was read at $450 \mathrm{~nm}$. All sera were tested in duplicate. The cut-off value was determined by the mean $\mathrm{OD}_{450}$ value of the 23 healthy sera sample plus two standard deviations. The sensitivity (\%) of the method was determined by the percentage value of ELISA positive samples and true positive cases, while the specificity (\%) was determined by that of ELISA negative samples and true negative cases. Sera from patients with cysticercosis (26 samples) were used to determine the cross-reactivity of $\mathrm{r} E g \mathrm{HK}$.

\section{Statistics analysis}

SPSS 19.0 software (IBM Corp., Armonk, NY, USA) was used for statistics analysis. The ELISA serological result was analysed using the nonparametric Kruskal-Wallis $\mathrm{H}$-test. The results were considered statistically significant at $P<0.05$.

\section{Results}

\section{Sequencing and bioinformatics analysis of $E g \mathrm{HK}$}

The full-length cDNA sequence of $E g \mathrm{HK}$ comprised 1395 nucleotides encoding 464 amino acids (aa). The predicted molecular weight and pI was $51.7 \mathrm{kDa}$ and 6.12, respectively. The instability index was 38.72 , lower than the threshold value (40), indicating that $E g \mathrm{HK}$ was a stable protein. There was no predicted transmembrane region in the deduced amino acid sequence. Analysis by SignalP showed that the deduced protein had no signal peptide. $E g \mathrm{HK}$ had a highly conserved active polypeptide chain (LGFTYSFPCEQAGLNTSFHVRWTKGF, 143-168 aa), which is the signature acid sequence of HK [27] (Fig. 1). There were 17 putative B-cell linear epitopes in the amino acid sequence of $E g \mathrm{HK}$, nine of which (ep1:6272 aa; ep2:93-102 aa; ep3:111-115 aa; ep4:186-191 
aа; ep5:248-256 aа; ep6:289-296 aа; ep7:343-352 aа; ep8:370-375 aa; ep9:448-456 aa), according to the comparison with the homologous human B-cell epitopes, suggested promising diagnostic and vaccine potentials. The putative glucose binding sites involve the following amino acids: Ser144, Phe145, Pro146, Thr161, Lys162, Asn196, Asp197, Leu217, Gly221, Thr222, Asn223, Glu249, Gln280 and Glu283 [27] (Fig. 1), all of which are conserved in $E g \mathrm{HK}$.

\section{Homologous sequence alignment}

Homologous sequence alignment revealed that EgHK shared $95.65 \%$ identity with HK from Echinococcus multilocularis (EmHK), 89.66\% identity with Hymenolepis microstomia (HmHK), 60.58\% identity with C. sinensis $(C s \mathrm{HK})$ and $56.98 \%$ identity with $S$. mansoni (SmHK). Moreover, EgHK shared 39.68, 40.95, 37.72 and 34.91\% identity with Homo sapiens HK-1, HK-2, HK-3 and HK-4, respectively. The phylogenetic tree revealed that $E g \mathrm{HK}$ was clustered into a branch closely related to $\mathrm{HK}$ from other cestode parasites, such as E. multilocularis and $H$. microstomia, and had the closest genetic relationship with E. multilocularis (Fig. 2).

\section{Expression, purification and identification of $\mathrm{rEgHK}$}

$E g \mathrm{HK}$ was expressed as an insoluble protein and existed in an inclusion body in E. coli BL21 (DE3) as a His-tag protein (Fig. 3). Purified rEgHK was detected by SDSPAGE and approximately presented the expected molecular weight of $51.7 \mathrm{kDa}$. The anti-sera against $\mathrm{rEgHK}$ exhibited a high titer $(1: 51,200)$. Recombinant $E g \mathrm{HK}$ was recognized by mouse anti-sera against $\mathrm{r} E g \mathrm{HK}$ and the sera from patients with CE (Fig. 3), while it was not recognized by pre-immune mouse sera or sera from healthy subjects.

Immunolocalization of EgHK in larval stage of E. granulosus To detect the localization of $E g \mathrm{HK}$ in the larval stage of $E$. granulosus, immunofluorescence assay was performed on E. granulosus protoscoleces and cyst walls using mouse anti-rEgHK sera. The results showed strong fluorescence intensity on the tegument surface, suckers and hooklets of protoscoleces (Fig. 4). Also, the entire cyst wall, including the germinal layer (GL) and laminated layer (LL), exhibited green fluorescence. No specific fluorescence was detected in the negative controls.

\section{Serology assay by rEgHK}

To evaluate the serodiagnostic potential of $\mathrm{rEgHK}$, the sera of patients with CE or Taenia solium cysticercosis and of healthy subjects were analyzed by indirect ELISA. The cut-off value calculated from the 23 samples collected from healthy subjects was 0.5 (Fig. 5). Forty-two serum samples from patients with $\mathrm{CE}$ were detected as positive for $\mathrm{r} E g \mathrm{HK}$, indicating a sensitivity of 91.3\% (42/46). The $\mathrm{OD}_{450}$ values of serum samples from patients with CE were significantly higher than those of patients with cysticercosis or healthy subjects (KruskalWallis H-test: $\left.\chi^{2}=63.571, d f=2, P<0.0001\right)$. The $\mathrm{OD}_{450}$ values of 20 serum samples from the healthy subjects and 23 samples from patients with cysticercosis were lower than the cut-off value, indicating a specificity of $87.8 \%$ $(43 / 49)$ in the assay. Moreover, three sera out of 26 samples from cysticercosis patients displayed cross-reactivity with $\mathrm{r} E g \mathrm{HK}$.

\section{Discussion}

In the study reported here, we analyzed the sequence, structure, immunoreactivity, immunogenicity and tissue distribution of $E g \mathrm{HK}$ in the larval stage of E. granulosus. We also evaluated the serodiagnostic potential of $E g \mathrm{HK}$ in CE patients.

In mammals, the HK family consists of three 100$\mathrm{kDa}$ isozymes (HK-1, HK-2, and HK-3) and one $50-\mathrm{kDa}$ isozyme (HK-4), also referred to as glucokinase. However, non-mamalian organisms generally have a $50-\mathrm{kDa}$ HK. It has been postulated that the $100-\mathrm{kDa}$ hexokinases evolved through the duplication and tandem ligation of a glucokinase-like gene that encodes an ancestral $50-\mathrm{kDa}$ HK [28]. The analysis of the amino acid squence showed that there was a single form of HK in E. granulosus and that $E g \mathrm{HK}$ possesses the typical characteristics of a nonmammalian HK (Fig.1). When subjected to SDS-PAGE, the molecular mass of $\mathrm{r} E g \mathrm{HK}$ was approximately 51.7 $\mathrm{kDa}$ (Fig. 3), which is the same as that described for HK from other parasites, such as P. falciparum, S. mansoni, and $C$. sinensis $[11,22,25]$.

As the first key enzyme in the glycolytic pathway, HK begins glycolysis and catalyzes the phosphorylation of glucose to produce glucose 6-phosphate. Previous gene expression profile has shown that $\mathrm{HK}$ is consistantly expressed in the oncospheres and in the larval and adult stages of E. granulosus. Furthermore, HK is upregulated in protoscoleces, the hydatid cyst and especially in adult worms compared with oncospheres, suggesting that HK plays important roles in controlling and maintaining stage-specific features of the parasite during its lifecycle [5]. Thus, the crucial importance of glycolysis to $E$. granulosus and the lower identity (34.91\%) of EgHK with human glucokinase (HK-4) suggest that this molecule could be a promising target for both chemotherapy and vaccine development.

The immunofluorescence study demonstrated that $E g \mathrm{HK}$ is extensively distributed in protoscoleces and the cyst wall, where protoscoleces are formed, suggesting 


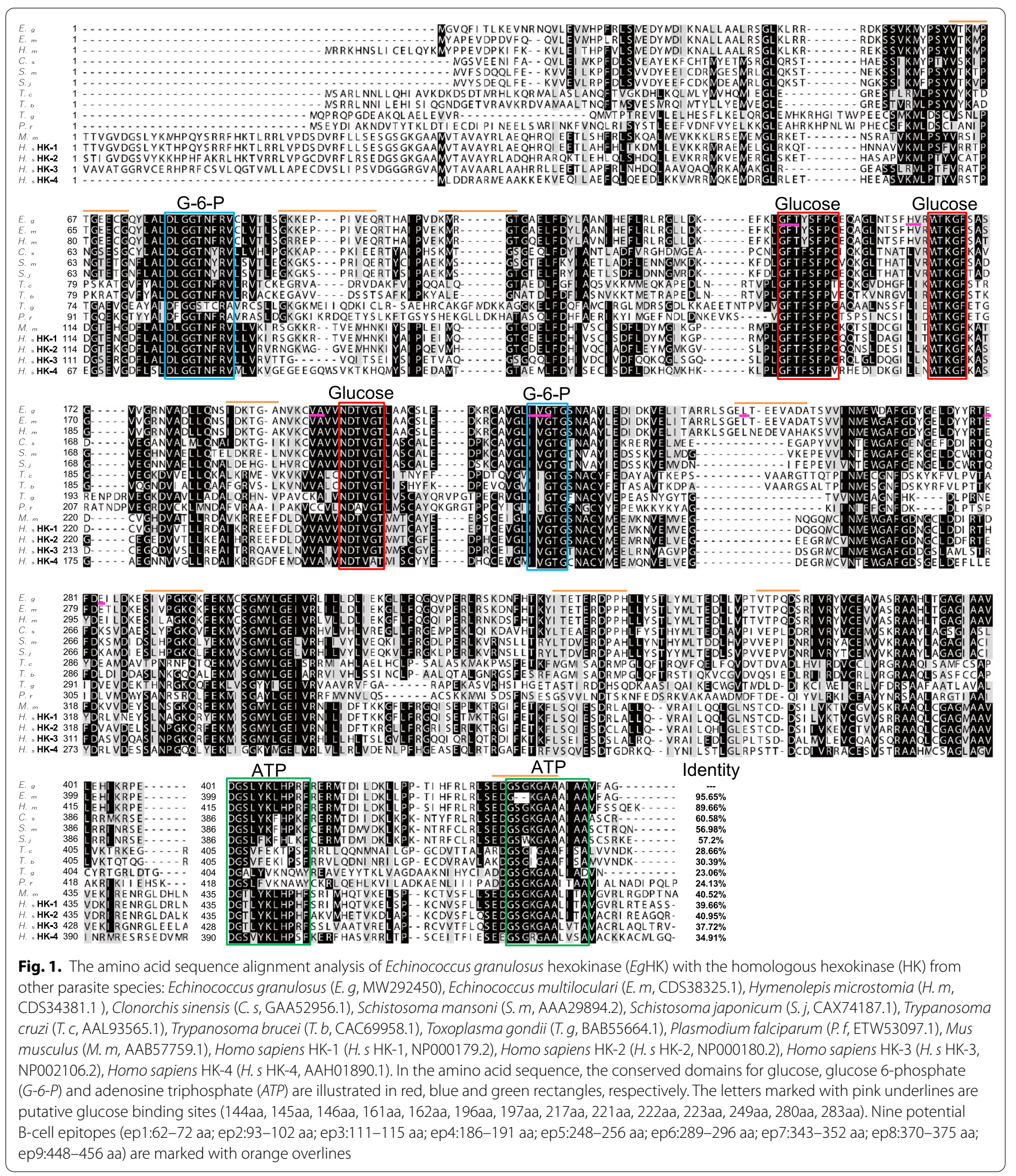

that as a key glycolytic enzyme, $E g \mathrm{HK}$ plays a vital role in the formation of the GL and the growth of protoscoleces. This conclusion is also supported by previous observations of high levels of $E g \mathrm{HK}$ mRNA and protein in the larval stage of E. granulosus [5, 29]. In protoscoleces, in particular, $E g \mathrm{HK}$ was observed to be abundantly expressed in the tegument. It is well known that the tegument of cestode is closely associated with the 


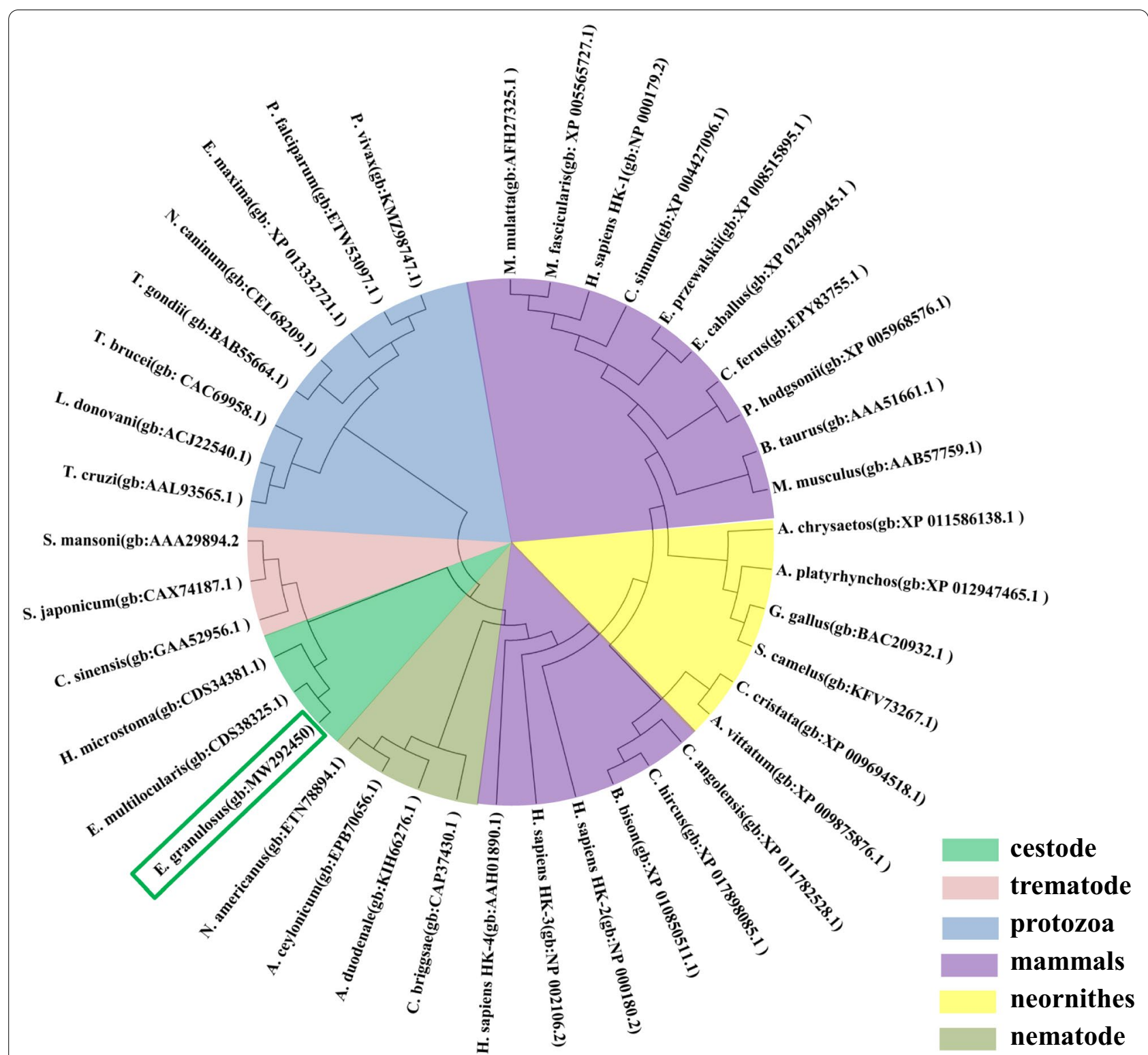

Fig. 2. The phylogenetic analysis of EgHK with homologous HK. The phylogenetic tree was constructed using the neighbor-joining method. $E$. granulosus, Echinococcus granulosus; E. multilocularis, Echinococcus multilocularis; H. microstoma, Hymenolepis microstomia; C. sinensis, Clonorchis sinensis; S. japonicum, Schistosoma japonicum; S. mansoni, Schistosoma mansoni; T. cruzi, Trypanosoma cruzi; L. donovani, Leishmania donovani; T. brucei, Trypanosoma brucei; T. gondii, Toxoplasma gondii; N. caninum, Neospora caninum; E. maxima, Eimeria maxima; P. falciparum, Plasmodium falciparum; P. vivax, plasmodium vivax; M. mulatta, Macaca mulatta; M. fascicularis, Macaca fascicularis; C. simum, Ceratotherium simum; E. przewalskii, Equus przewalskii; E. caballus, Equus caballus; C. ferus, Camelus ferus; P. hodgsonii, Pantholops hodgsonii; B. taurus, Bos Taurus; M. musculus, Mus musculus; A. chrysaetos, Aquila chrysaetos; A. platyrhynchos, Anas platyrhynchos; G. gallus, Gallus gallus; S. camelus, Struthio camelus; C. cristata, Cariama cristata; A. vittatum, Apaloderma vittatum; C. angolensis, Colobus angolensis; C. hircus, Capra hircus; B. bison, Bison bison; H. sapiens, Homo sapiens; C. briggsae, Caenorhabditis briggsae; A. duodenale, Ancylostoma duodenale; A. ceylanicum, Ancylostoma ceylonicum; N. americanus, Necator americanus. gb GenBank ID

absorption of nutrients. Thus, the distribution of $E g \mathrm{HK}$ in the tegument indicates its pivotal role in the absorption and digestion of glucose from the host for energy supply. Furthermore, the localization of $E g \mathrm{HK}$ in the suckers and hooklets of protoscoleces might be associated with the energy requirement for the process of attaching to the host intestine. The cyst wall, which is composed of the GL and LL, is the parasite-host interface and is permeable; as such, it is believed to be involved in the parasite-host interaction, enabling the diffusion of macromolecules of 


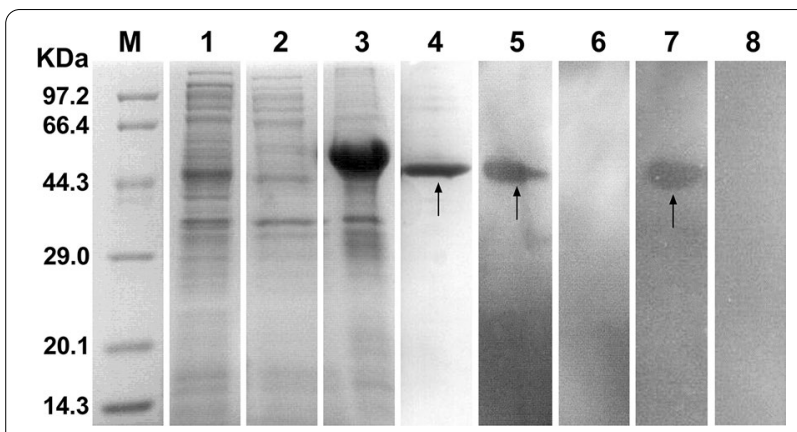

Fig. 3. Expression, purification and western blot analysis of $\mathrm{rEgHK}$. Lanes: M Molecular marker (in KDa), 1 induced E. coli BL21 (DE3) transformed with pET30a, 2 soluble supernatant fraction of induced E. coli BL21 (DE3) transformed with pET30a-EgHK, 3 pellet fraction of induced E. coli BL21 (DE3) transformed with pET30a-EgHK, 4 purified $\mathrm{rEgHK}, 5$ purified $\mathrm{rEgHK}$ probed with anti-rEgHK sera, 6 purified $\mathrm{rEgHK}$ probed with pre-immunized sera, 7 purified $\mathrm{rEgHK}$ probed with sera from patient with $\mathrm{CE}, 8$ purified $\mathrm{rEgHK}$ probed with sera from healthy subjects

at least $150 \mathrm{kDa}$ [30]. The outer, acellular LL, in particular, is widely regarded as being a crucial element of parasite-host interplay [31]. Given that $E g H K$ was detected in not only the GL, but also in the acellular LL, the localization of $E g \mathrm{HK}$ in the metacestode suggests that $E g \mathrm{HK}$ might be a component of excretory/secretory (ES) products and mediate direct interaction with host cells.

In our study, the high titer of anti-rEgHK antibody IgG demonstrates the strong immunogenicity of $\mathrm{rEgHK}$. In addition, $\mathrm{r} E g \mathrm{HK}$ was recognized by anti-rEgHK mouse sera and the sera from patients with CE, demonstrating its good immunoreactivity. In particular, $\mathrm{r} E g \mathrm{HK}$ recognition by the sera from patients with $\mathrm{CE}$ suggests that $E g \mathrm{HK}$ might be a component of ES products of the E. granulosus metacestode, which is consistent with previous conjectures. However, the predicted amino acid sequence of $E g H K$ contains no signal peptide or transmembrane domain. Thus, we speculate that $E g \mathrm{HK}$ may rely on the release of exosomes, a special protein secretion pathway that has been reported in E. granulosus [32], for extracellular secretion.

At present, the commercially available serological kits mostly use hydatid cyst fluid (HCF) as diagnostic antigen, collected from infected animals. However, the complex and heterogeneous composition of HCF negatively impacts on the sensitivity and specificity of the tests [33, 34]. Furthermore, there is a growing evidence indicating that recombinant proteins and high-purity synthetic antigen preparations are more reliable for serodiagnostic application than native antigens. Thus, research on recombinant proteins and synthetic peptides is an important strategy to develop more sensitive and specific tests [35]. As the first enzyme in glycolysis, $E g \mathrm{HK}$, which is crucial to E. granulosus, has been identified as a potential antigenic protein [29]. Therefore, in this study, we evaluated the diagnostic value of $\mathrm{r} E g \mathrm{HK}$ in patients with $\mathrm{CE}$ using an indirect ELISA. The sensitivity and specificity of the $\mathrm{r} E g \mathrm{HK}$ assay achieved $91.3 \%$ and $87.8 \%$, respectively, in the pilot serological assay, reflecting its potential applications. Nevertheless, rEgHK showed cross-reactions with three serum samples out of 26 samples of sera from patients with T. solium cysticercosis, indicating that EgHK protein shares a few of the same or similar epitopes with T. solium proteins. This is inevitable considering

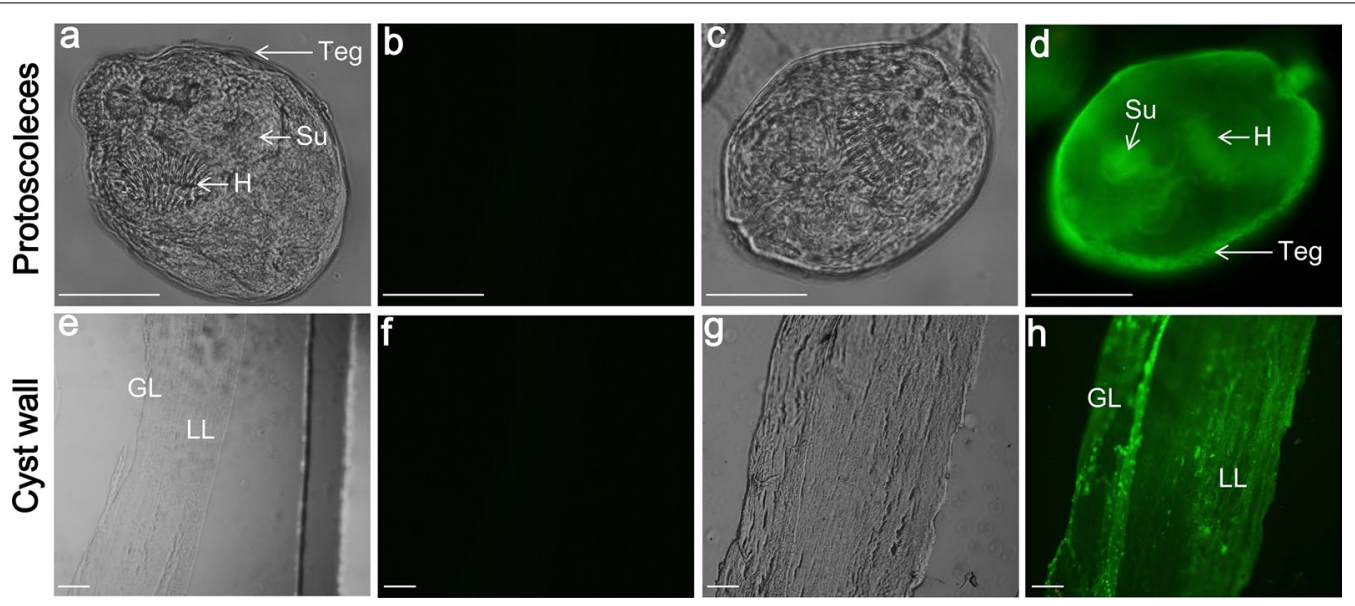

Fig. 4. Immunolocalization of EgHK in larval stage of E. granulosus. EgHK in the protoscoleces and the cyst walls was immunofluorescently labeled using mouse anti-rEgHK sera as the primary antibody, followed by fluorescein isothiocyanate-conjugated anti-mouse immunoglobulin $\mathrm{G}$ as secondary antibody. Sera from pre-immune mice were applied as primary antibody for a negative control. $\mathbf{b}, \mathbf{d}, \mathbf{f}, \mathbf{h}$ are fluorescence microscopy images; $\mathbf{a}, \mathbf{c}, \mathbf{e}, \mathbf{g}$ are the respective images of the same samples observed under white light. Teg Tegument, Su sucker, $H$ hooklet, $L L$ laminated layer, GL germinal layer. Scale bars: $50 \mu \mathrm{m}$ 


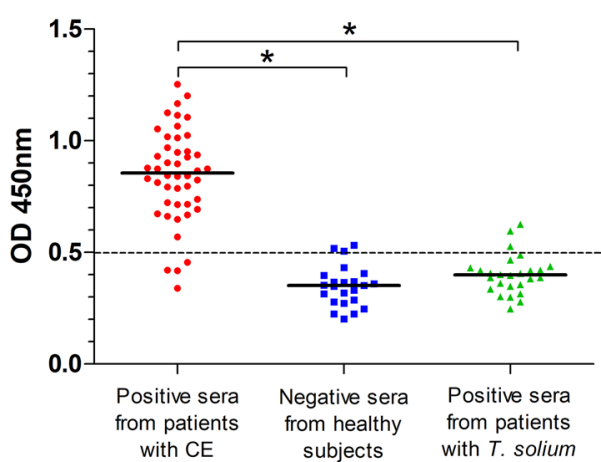

Fig. 5. Serological results from the enzyme-linked immunosorbent assay for sera from patients with cystic echinococcosis (CE) or Taenia solium cysticercosis and from healthy, negative subjects for EgHK. Horizontal black lines represent the median values. Dashed horizontal line indicates the cut-off value (0.5). Asterisk indicates a signficant difference at $P<0.0001$.

their close genetic relationship. Analysis of the crossreaction of $\mathrm{r} E g \mathrm{HK}$ with $E$. multilocularis was not performed due to the lack of serum samples from alveolar echinococcosis patients. Therefore, further studies are needed to validate the clinical applicability of $\mathrm{r} E g \mathrm{HK}$ in the future.

\section{Conclusions}

We report here the bioinformatic characterization of $E g \mathrm{HK}$. Immunofluorescence assay verified that $E g \mathrm{HK}$ is mainly expressed on the tegument, suckers and hooklets of protoscoleces and on the cyst wall. The recombinant $E g \mathrm{HK}$ showed relatively good immunogenicity and immunoreactivity. The indirect $\mathrm{rEgHK}$-ELISA indicated a good sensitivity (91.3\%) and specificity (87.8\%) for the detection of antibodies in sera from patients with CE. Our results suggest that $E g \mathrm{HK}$ may be a promising candidate for development of vaccines against E. granulosus and an effective antigen for the diagnosis of human $\mathrm{CE}$.

\section{Abbreviations}

CE: cystic echinococcosis; HK: hexokinase; EgHK: E. granulosus HK; rEgHK: recombinant EgHK; cDNA: complementary DNA; aa: amino acids; GL: germinal layer; LL: laminated layer; ELISA: enzyme linked immunosorbent assay.

\section{Acknowledgements}

Not applicable.

\section{Authors' contributions}

TJ conceived this project, designed the experiment and reviewed the manuscript. QX and MY designed and performed the experiment, analyzed and interpreted the data and drafted the manuscript. WL, HL, XS and JL participated in performing the experiment. All authors read and approved the final manuscript.

\section{Funding}

This research was supported by the Fundamental Research Funds for the Central Universities (No. Izujbky-2020-50), Natural Science Foundation of Gansu Province (No. 20JR10RA636).

\section{Availability of data and materials}

The full-length DNA sequence of EgHK has been deposited in GenBank database (https://www.ncbi.nlm.nih.gov/) under the accession number MW292450. Data supporting the conclusions of this article are included within the article. The data used and/or analyzed during the current study are available from the corresponding author upon reasonable request.

\section{Ethics approval and consent to participate}

This study protocol was approved by the Research Ethics Committee and the Animal Caring and Using Committee of Lanzhou University.

\section{Consent for publication}

Not applicable.

\section{Competing interests}

The authors declare that they have no competing interests.

\section{Author details}

${ }^{1}$ Institute of Pathogenic Biology, School of Basic Medical Sciences, Lanzhou University, Lanzhou 730000, Gansu, People's Republic of China. ${ }^{2}$ The Eighth Affiliated Hospital, Sun Yat-sen University, Shenzhen 518000, Guangdong, People's Republic of China.

Received: 30 September 2020 Accepted: 23 January 2021 Published online: 08 February 2021

References

1. Cadavid Restrepo AM, Yang YR, McManus DP, Gray DJ, Giraudoux P, Barnes TS, et al. The landscape epidemiology of echinococcoses. Infect Dis Poverty. 2016;5:13.

2. Torgerson PR, de Silva NR, Fèvre EM, Kasuga F, Rokni MB, Zhou XN, et al. The global burden of foodborne parasitic diseases: an update. Trends Parasitol. 2014;30:20-6.

3. Budke CM, Deplazes P, Torgerson PR. Global socioeconomic impact of cystic echinococcosis. Emerg Infect Dis. 2006;12:296-303.

4. World Health Organization and World Organisation for Animal Health. Report of the WHO informal working group on cystic and alveolar echinococcosis surveillance, prevention and control, with the participation of the Food and Agriculture Organization of the United Nations and the World Organisation for Animal Health. 2011. http://apps.who.int/iris/bitst ream/10665/44785/1/9789241502924_eng.pdf. Accessed 15 Dec 2014.

5. Zheng H, Zhang W, Zhang L, Zhang Z, Li J, Lu G, et al. The genome of the hydatid tapeworm Echinococcus granulosus. Nat Genet. 2013;45:1 168-75.

6. Agosin M. Studies on the metabolism of Echinococcus granulosus. II. Some observations on the carbohydrate metabolism of hydatid cyst scolices. Exp Parasitol. 1957:6:586-93.

7. McManus DP, Smyth JD. Differences in the chemical composition and carbohydrate metabolism of Echinococcus granulosus (horse and sheep strains) and E. multilocularis. Parasitology. 1978;77:103-9.

8. Lorenzatto KR, Monteiro KM, Paredes R, Paludo GP, da Fonsêca MM, Galanti N, et al. Fructose-bisphosphate aldolase and enolase from Echinococcus granulosus: genes, expression patterns and protein interactions of two potential moonlighting proteins. Gene. 2012;506:76-84.

9. Wu M, Yan M, Xu J, Yin X, Dong X, Wang N, et al. Molecular characterization of triosephosphate isomerase from Echinococcus granulosus. Parasitol Res. 2018;117:3169-76.

10. Bodmer WF. The enzymes of glycolysis: structure, activity and evolution. Philos Trans R Soc Lond B Biol Sci. 1981;293:1-214.

11. Olafsson P, Matile $H$, Certa U. Molecular analysis of Plasmodium falciparum hexokinase. Mol Biochem Parasitol. 1992;56:89-101. 
12. Tjhin ET, Staines HM, van Schalkwyk DA, Krishna S, Saliba KJ. Studies with the Plasmodium falciparum hexokinase reveal that PfHT limits the rate of glucose entry into glycolysis. FEBS Lett. 2013;587:3182-7.

13. Harris MT, Walker DM, Drew ME, Mitchell WG, Dao K, Schroeder CE, et al. Interrogating a hexokinase-selected small-molecule library for inhibitors of Plasmodium falciparum hexokinase. Antimicrob Agents Chemother. 2013;57:3731-7.

14. Pabón MA, Cáceres AJ, Gualdrón M, Quiñones W, Avilán L, Concepción J. Purification and characterization of hexokinase from Leishmania mexicana. Parasitol Res. 2007;100:803-10.

15. Willson M, Sanejouand YH, Perie J, Hannaert V, Opperdoes F. Sequencing, modeling, and selective inhibition of Trypanosoma brucei hexokinase. Chem Biol. 2002:9:839-47.

16. Sharlow ER, Lyda TA, Dodson HC, Mustata G, Morris MT, Leimgruber SS, et al. A target-based high throughput screen yields Trypanosoma brucei hexokinase small molecule inhibitors with antiparasitic activity. PLoS Negl Trop Dis. 2010;4:e659.

17. Cáceres AJ, Portillo R, Acosta H, Rosales D, Quiñones W, Avilan L, et al. Molecular and biochemical characterization of hexokinase from Trypanosoma cruzi. Mol Biochem Parasitol. 2003;126:251-62.

18. Hudock MP, Sanz-Rodríguez CE, Song Y, Chan JM, Zhang Y, Odeh S, et al. Inhibition of Trypanosoma cruzi hexokinase by bisphosphonates. J Med Chem. 2006;49:215-23.

19. Sanz-Rodríguez CE, Concepción JL, Pekerar S, Oldfield E, Urbina JA. Bisphosphonates as inhibitors of Trypanosoma cruzi hexokinase: kinetic and metabolic studies. J Biol Chem. 2007;282:12377-87.

20. Schmitt-Wrede HP, Waldraff A, Krücken J, Harder A, Wunderlich F. Characterization of a hexokinase encoding cDNA of the parasitic nematode Hhaemonchus contortus. Biochim Biophys Acta. 1999;1444:439-44.

21. Singh AR, Joshi S, Arya R, Kayastha AM, Srivastava KK, Tripathi LM, et al. Molecular cloning and characterization of Brugia malayi hexokinase. Parasitol Int. 2008;57:354-61.

22. Tielens AG, van den Heuvel JM, van Mazijk HJ, Wilson JE, Shoemaker CB. The 50-kDa glucose 6-phosphate-sensitive hexokinase of Schistosoma mansoni. J Biol Chem. 1994;269:24736-41.

23. Shoemaker CB, Reynolds SR, Wei G, Tielens AG, Harn DA. Schistosoma mansoni hexokinase: CDNA cloning and immunogenicity studies. Exp Parasitol. 1995;80:36-45.

24. Armstrong RL, Wilson JE, Shoemaker CB. Purification and characterization of the hexokinase from Schistosoma mansoni, expressed in Escherichia coli. Protein Expr Purif. 1996;8:374-80.
25. Chen T, Ning D, Sun H, Li R, Shang M, Li X, et al. Sequence analysis and molecular characterization of Clonorchis sinensis hexokinase, an unusual trimeric 50-kDa glucose-6-phosphate-sensitive allosteric enzyme. PLoS One. 2014;9:e107940.

26. Chen T, Yu J, Tang Z, Xie Z, Lin Z, Sun H, et al. Advanced enzymology, expression profile and immune response of Clonorchis sinensis hexokinase show its application potential for prevention and control of clonorchiasis. PLoS Negl Trop Dis. 2015;9:e0003641.

27. Mulichak AM, Wilson JE, Padmanabhan K, Garavito RM. The structure of mammalian hexokinase-1. Nat Struct Biol. 1998;5:555-60.

28. Wilson JE. Hexokinases. Rev Physiol Biochem Pharmacol. 1995;126:65-198.

29. Cui SJ, Xu LL, Zhang T, Xu M, Yao J, Fang CY, et al. Proteomic characterization of larval and adult developmental stages in Echinococcus granulosus reveals novel insight into host-parasite interactions. J Proteomics. 2013:84:158-75.

30. Coltorii EA, Varela-Díaz VM. Echinococcus granulosus: penetration of macromolecules and their localization on the parasite membranes of cysts. Exp Parasitol. 1974;35:225-31.

31. Díaz A, Casaravilla C, Allen JE, Sim RB, Ferreira AM. Understanding the laminated layer of larval Echinococcus II: immunology. Trends Parasitol. 2011;27:264-73.

32. Siles-Lucas M, Sánchez-Ovejero C, González-Sánchez M, González E, Falcón-Pérez JM, Boufana B, et al. Isolation and characterization of exosomes derived from fertile sheep hydatid cysts. Vet Parasitol. 2017;236:22-33.

33. Frayha GJ, Haddad R. Comparative chemical composition of protoscolices and hydatid cyst fluid of Echinococcus granulosus (Cestoda). Int J Parasitol. 1980;10:359-64.

34. Ahn CS, Han X, Bae YA, Ma X, Kim JT, Cai H, et al. Alteration of immunoproteome profile of Echinococcus granulosus hydatid fluid with progression of cystic echinococcosis. Parasites Vectors. 2015:8:10.

35. Manzano-Román R, Sánchez-Ovejero C, Hernández-González A, Casulli A, Siles-Lucas M. Serological diagnosis and follow-up of human cystic echinococcosis: a new hope for the future? Biomed Res Int. 2015;2015:428205

\section{Publisher's Note}

Springer Nature remains neutral with regard to jurisdictional claims in published maps and institutional affiliations.
Ready to submit your research? Choose BMC and benefit from:

- fast, convenient online submission

- thorough peer review by experienced researchers in your field

- rapid publication on acceptance

- support for research data, including large and complex data types

- gold Open Access which fosters wider collaboration and increased citations

- maximum visibility for your research: over $100 \mathrm{M}$ website views per year

At BMC, research is always in progress.

Learn more biomedcentral.com/submissions 